\title{
Iris Recognition Using Ridgelets
}

\author{
Lenina Birgale* and Manesh Kokare**
}

\begin{abstract}
Image feature extraction is one of the basic works for biometric analysis. This paper presents the novel concept of application of ridgelets for iris recognition systems. Ridgelet transforms are the combination of Radon transforms and Wavelet transforms. They are suitable for extracting the abundantly present textural data that is in an iris. The technique proposed here uses the ridgelets to form an iris signature and to represent the iris. This paper contributes towards creating an improved iris recognition system. There is a reduction in the feature vector size, which is $1 \mathrm{X} 4$ in size. The False Acceptance Rate (FAR) and False Rejection Rate (FRR) were also reduced and the accuracy increased. The proposed method also avoids the iris normalization process that is traditionally used in iris recognition systems. Experimental results indicate that the proposed method achieves an accuracy of $99.82 \%, 0.1309 \%$ FAR, and $0.0434 \%$ FRR.
\end{abstract}

Keywords—Ridgelets, Texture, Wavelets, Biometrics, Features, Database

\section{INTRODUCTION}

Security systems are the need of the day. Efficient and fast recognition systems are the demand of the current era. Facial features, voice patterns, hand geometry, retinal patters, vein patterns, signature dynamics, voice verification, facial thermographs, DNA matching nail-bed identification, gait recognition, ear shape recognition, and finger prints have all been explored as biometric identifiers with varying levels of success. However irises, which are unique and stable for the duration of a person's life, have emerged as the most reliable biometric. Irises as a biometric for identification have been an active research area since 1992. The uniqueness of iris patterns was identified by A. Muron and J. Pospisil, [1-5]. This uniqueness property of irises can be explained in the words of Daugman $[6,7]$ as, "An advantage that the iris shares with finger prints is the chaotic morphogenesis of its minutiae." The iris can be identified as the colored portion of the eye lying between the pupil and sclera. A frontal view of the human eye is shown in Fig. 1. A very important characteristic of an iris is that it is a naturally protected organ and is stable without any variations including effects of an individual aging. It is the source of abundant textural information. This texture has to be exploited completely for a better biometric system design. Traditional methods are computationally expensive. Hence the challenge in an iris recognition system is to develop a fast responding and computationally inexpensive system for online applications. The reduction of computational complexity, without losing track of other system performance parameters, motivates us to solve this problem [1]. Though wavelets detect point singularities, they are computationally expensive as demonstrated by Terrades and

\footnotetext{
Manuscript received Octorber 27, 2011; accepted March 8, 2012.

Corresponding Author: Lenina Birgale

* Dept. of Electronics and Telecommunication Engineering, Shri Guru Gobind Singhji Institute of Engineering and Technology, Vishnupuri, Nanded, Maharashtra State, India (lenina2003_2003@yahoo.com, mbkokare@sggs.ac.in)
} 


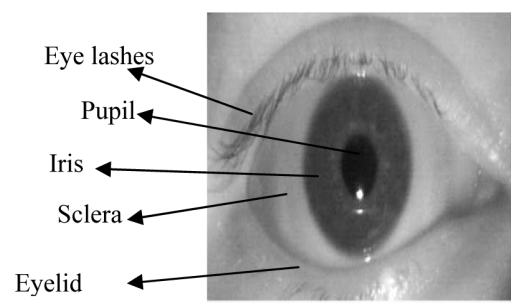

Fig. 1. A frontal view of the human eye

Valveny [8]. Different sub bands of multilevel decomposition in wavelets lengthen the iris signature size. These lengthy signatures in turn increase the computational complexity. Long signatures hamper system performance. This has also been a matter of concern in the previous bodies of work [9-13]. Wavelets are very good at representing point singularities, but when it comes to line singularities wavelets fail and ridgelets are the solution. The basic property of ridgelets for detecting line singularities has been our motivation for selecting ridgelets as a tool.

In this paper we propose a simple and efficient algorithm based on the ridgelet for iris recognition, which combines the masking process with a ridgelet transform for feature extraction, in order to represent an iris.

The remainder of this paper is organized as follows: in the next section, related work and the theoretical background about the iris as a biometric is presented. Proposed masks are discussed in Section 3. A brief overview of ridgelets, Radon transforms, and Discrete Wavelet Transforms are presented in Section 4. Section 5 gives the proposed method. Section 6 and Section 7 present the experimental results and conclusions, respectively.

\section{RELATED WORK}

Various research groups such as [14-26], have proposed an iris recognition system based on iris normalization. The iris normalization step has become so popular in the research groups that it is being called Daugman's 'Rubber Sheet Model' after renowned researcher J. Daugman. There are a number of methods for iris recognition. To mention a few very recent ones there are [2-5, 16, 24, 26-41]. Almost all of these methods use iris normalization. The iris signature of these methods is of significant length. The novelty of the proposed method starts right from the step of normalization. Iris segmentation and its normalization are shown in Fig. 2. Our work completely avoids iris normalization. Most of the researchers use the Chinese Academy of Sciences-Institute of Automation-CASIA database [42]. The database used here is also from the CASIA database. In addition, the UPOL database is also used. This work shows that ridgelets provide the abundant texture information that is present in an iris for improving the system performance in terms of accuracy, False Acceptance Rate, False Rejection Rate, and speed.

The proposed method gives improvement in the accuracy, reduction in FAR and FRR even with reduced signature size. It uses ridgelets which is the combination of Radon Transform and Wavelets for information extraction required in iris signature formation. Here the proposed method has used single level, wavelet decomposition. Higher level decomposition introduces redundancy. Single level decomposition gives better results. Obtained results are compared with the previous method $[6-7,14-15,19,21,29]$. All these earlier methods use iris normaliza- 

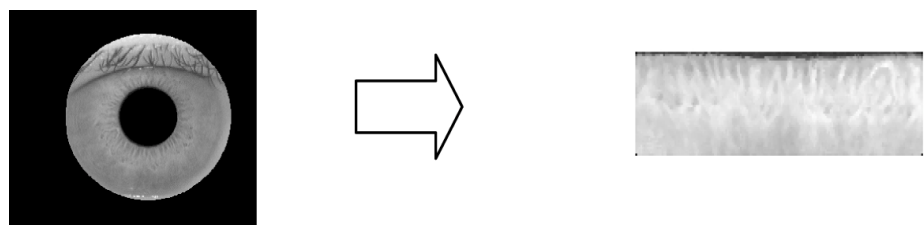

Fig. 2. The results of image after segmentation and normalization

tion , and iris signature of significant length. The proposed method uses mask to avoid iris normalization. Iris signature used here is just of size $1 \mathrm{X} 4$. The proposed iris signature is comparatively very small but efficient enough to represent iris features.

\subsection{Main Contribution}

Any biometric system is expected to function with minimum feature signature, reduced FAR, reduced FRR and highest accuracy. To achieve all these requirements the authors use the Ridgelet transform. The main contributions of this paper are summarized as follows. The proposed method, firstly, reduces the iris signature size to improve system speed. Ridgelets being the combination of Radon Transform and Wavelet Transform, relate scales of line positions. These line scales extract the abundant texture from an iris and give maximum unique information with minimum signature size. The reduced signature size thus improves the system speed without paying any significant cost in terms of accuracy. Secondly, proposed method skips the process of iris normalization, which contributes towards significant reduction in processing time and thus improving the system performance. Thirdly, the ridgelet transform used in the proposed method have increased accuracy rate, and reduced the False Acceptance Rate and the False Rejection Rate significantly. Finally, this work compares the obtained results with few of the existing methods.

\section{PROPOSED IRIS MASK}

As mentioned in the earlier section, major hurdles for computational efficiency are normalization and quantization. Eyelid and eyelash occlusions form the major noise component in an iris recognition system. They need careful attention paid to them in order to minimize the FAR and FRR. Basically eyelid and eyelash occlusions cause major disturbances in partially opened eyes from the upper and lower side of an iris as shown in Fig. 3(1). Figs. 3(2) and 3(3) show that eyelid and eyelash occlusions can be avoided by the use of a proper mask for segmentation. The use of an appropriate mask avoids unnecessary processing. There is definitely an opposite effect of this process of using the mask. That is the iris region obtained for processing is reduced. However, even if this region considered for signature formation it hardly gives any actual iris patterns. We used the Discrete Wavelet Transform (DWT) for feature extraction. The mask equation is designed by using equation (1), (2) and is given by equation (3).

$$
A_{I P}=\sum_{\mathrm{N}=0}^{259} \sum_{r_{i}=1}^{R_{\operatorname{Im} a x}} I\left(r_{i} \cos \left(\frac{2 \pi N}{N}\right)+P_{C C}, r_{i} \sin \left(\frac{2 \pi N}{N}\right)+P_{C R}\right)
$$




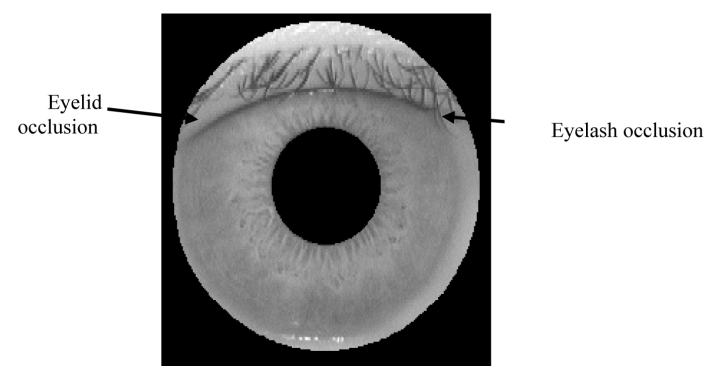

(1) Segmented iris with eyelid and eyelash occlusions

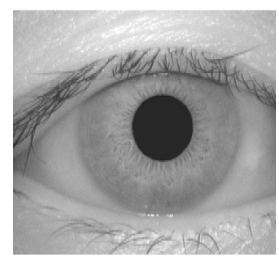

(2) Original iris image with eyelid and eyelash occlusions

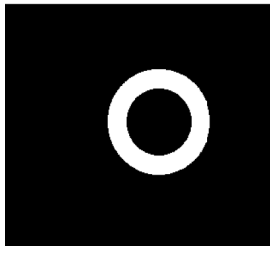

(3) Designed mask image

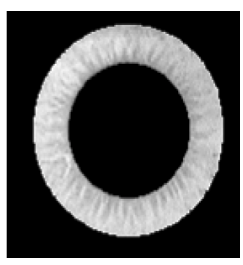

(4) Segmented iris without eyelid and eyelash occlusions

Fig. 3. Process of iris segmentation using a mask

$$
\begin{gathered}
A_{P}=\sum_{N=0}^{259} \sum_{r_{p}=1}^{R_{P m} a x} I\left(r_{p} \cos \left(\frac{2 \pi N}{N}\right)+P_{C C}, r_{p} \sin \left(\frac{2 \pi N}{N}\right)+P_{C R}\right) \\
\text { Mask }=A_{I P}-A_{P}
\end{gathered}
$$

Where:

$A_{I P}$ is the area of the iris and the pupil.

$A_{P}$ is the area of the pupil.

$R_{\text {Imax }}$ is the maximum radius of the iris.

$R_{P \max }$ is the maximum radius of the pupil.

$P_{C C}, P_{C R}$ are the coordinates of the centre of the pupil

$r_{i}$ is the initial radius of the iris.

$r_{p}$ is the initial radius of the iris.

Initial values of the radius are computed by noting the change in the intensity levels of the pupil and iris along the major and minor axis of the pupil. As the pupil is assumed to be elliptical, its centre is computed to be the intersection of the major and minor axis.

Fig. 4 shows a scaled mask and its coefficients. This mask is scaled to an image size of $30 \times 30$ for representation. 


\begin{tabular}{|c|c|c|c|c|c|c|c|c|c|c|c|c|c|c|c|c|c|c|c|c|c|c|c|c|c|c|c|c|c|}
\hline & 0 & 0 & 0 & 0 & 0 & 0 & 0 & 0 & 0 & 0 & 0 & 0 & 0 & 0 & 0 & 0 & 0 & 0 & 0 & 0 & 0 & 0 & 0 & 0 & 0 & 0 & 0 & 0 & 0 \\
\hline 0 & 0 & 0 & 0 & 0 & 0 & 0 & 0 & 0 & 0 & 0 & 1 & 1 & 1 & 1 & 1 & 1 & 1 & 1 & 0 & 0 & 0 & 0 & 0 & 0 & 0 & 0 & 0 & 0 & 0 \\
\hline 0 & 0 & 0 & 0 & 0 & 0 & 0 & 0 & 0 & 1 & 1 & 1 & 1 & 1 & 1 & 1 & 1 & 1 & 1 & 1 & 1 & 0 & 0 & 0 & 0 & 0 & 0 & 0 & 0 & 0 \\
\hline 0 & 0 & 0 & 0 & 0 & 0 & 0 & 1 & 1 & 1 & 1 & 1 & 1 & 1 & 1 & 1 & 1 & 1 & 1 & 1 & 1 & 1 & 1 & 0 & 0 & 0 & 0 & 0 & 0 & 0 \\
\hline 0 & 0 & 0 & 0 & 0 & 0 & 1 & 1 & 1 & 1 & 1 & 1 & 1 & 1 & 1 & 1 & 1 & 1 & 1 & 1 & 1 & 1 & 1 & 1 & 0 & 0 & 0 & 0 & 0 & 0 \\
\hline 0 & 0 & 0 & 0 & 0 & 1 & 1 & 1 & 1 & 1 & 1 & 1 & 1 & 1 & 1 & 1 & 1 & 1 & 1 & 1 & 1 & 1 & 1 & 1 & 1 & 0 & 0 & 0 & 0 & 0 \\
\hline 0 & 0 & 0 & 0 & 1 & 1 & 1 & 1 & 1 & 1 & 1 & 1 & 1 & 1 & 1 & 1 & 1 & 1 & 1 & 1 & 1 & 1 & 1 & 1 & 1 & 1 & 0 & 0 & 0 & 0 \\
\hline 0 & 0 & 0 & 1 & 1 & 1 & 1 & 1 & 1 & 1 & 1 & 1 & 1 & 1 & 1 & 1 & 1 & 1 & 1 & 1 & 1 & 1 & 1 & 1 & 1 & 1 & 1 & 0 & 0 & 0 \\
\hline 0 & 0 & 0 & 1 & 1 & 1 & 1 & 1 & 1 & 1 & 1 & 1 & 0 & 0 & 0 & 0 & 0 & 0 & 1 & 1 & 1 & 1 & 1 & 1 & 1 & 1 & 1 & 0 & 0 & 0 \\
\hline 0 & 0 & 1 & 1 & 1 & 1 & 1 & 1 & 1 & 1 & 1 & 0 & 0 & 0 & 0 & 0 & 0 & 0 & 0 & 1 & 1 & 1 & 1 & 1 & 1 & 1 & 1 & 1 & 0 & 0 \\
\hline 0 & 0 & 1 & 1 & 1 & 1 & 1 & 1 & 1 & 1 & 0 & 0 & 0 & 0 & 0 & 0 & 0 & 0 & 0 & 0 & 1 & 1 & 1 & 1 & 1 & 1 & 1 & 1 & 0 & 0 \\
\hline 0 & 1 & 1 & 1 & 1 & 1 & 1 & 1 & 1 & 0 & 0 & 0 & 0 & 0 & 0 & 0 & 0 & 0 & 0 & 0 & 0 & 1 & 1 & 1 & 1 & 1 & 1 & 1 & 1 & 0 \\
\hline 0 & 1 & 1 & 1 & 1 & 1 & 1 & 1 & 0 & 0 & 0 & 0 & 0 & 0 & 0 & 0 & 0 & 0 & 0 & 0 & 0 & 0 & 1 & 1 & 1 & 1 & 1 & 1 & 1 & 0 \\
\hline 0 & 1 & 1 & 1 & 1 & 1 & 1 & 1 & 0 & 0 & 0 & 0 & 0 & 0 & 0 & 0 & 0 & 0 & 0 & 0 & 0 & 0 & 1 & 1 & 1 & 1 & 1 & 1 & 1 & 0 \\
\hline 0 & 1 & 1 & 1 & 1 & 1 & 1 & 1 & 0 & 0 & 0 & 0 & 0 & 0 & 0 & 0 & 0 & 0 & 0 & 0 & 0 & 0 & 1 & 1 & 1 & 1 & 1 & 1 & 1 & 0 \\
\hline 0 & 1 & 1 & 1 & 1 & 1 & 1 & 1 & 0 & 0 & 0 & 0 & 0 & 0 & 0 & 0 & 0 & 0 & 0 & 0 & 0 & 0 & 1 & 1 & 1 & 1 & 1 & 1 & 1 & 0 \\
\hline 0 & 1 & 1 & 1 & 1 & 1 & 1 & 1 & 0 & 0 & 0 & 0 & 0 & 0 & 0 & 0 & 0 & 0 & 0 & 0 & 0 & 0 & 1 & 1 & 1 & 1 & 1 & 1 & 1 & 0 \\
\hline 0 & 1 & 1 & 1 & 1 & 1 & 1 & 1 & 0 & 0 & 0 & 0 & 0 & 0 & 0 & 0 & 0 & 0 & 0 & 0 & 0 & 0 & 1 & 1 & 1 & 1 & 1 & 1 & 1 & 0 \\
\hline 0 & 1 & 1 & 1 & 1 & 1 & 1 & 1 & 1 & 0 & 0 & 0 & 0 & 0 & 0 & 0 & 0 & 0 & 0 & 0 & 0 & 1 & 1 & 1 & 1 & 1 & 1 & 1 & 1 & 0 \\
\hline 0 & 0 & 1 & 1 & 1 & 1 & 1 & 1 & 1 & 1 & 0 & 0 & 0 & 0 & 0 & 0 & 0 & 0 & 0 & 0 & 1 & 1 & 1 & 1 & 1 & 1 & 1 & 1 & 0 & 0 \\
\hline 0 & 0 & 1 & 1 & 1 & 1 & 1 & 1 & 1 & 1 & 1 & 0 & 0 & 0 & 0 & 0 & 0 & 0 & 0 & 1 & 1 & 1 & 1 & 1 & 1 & 1 & 1 & 1 & 0 & 0 \\
\hline 0 & 0 & 0 & 1 & 1 & 1 & 1 & 1 & 1 & 1 & 1 & 1 & 0 & 0 & 0 & 0 & 0 & 1 & 1 & 1 & 1 & 1 & 1 & 1 & 1 & 1 & 1 & 0 & 0 & 0 \\
\hline 0 & 0 & 0 & 1 & 1 & 1 & 1 & 1 & 1 & 1 & 1 & 1 & 1 & 1 & 1 & 1 & 1 & 1 & 1 & 1 & 1 & 1 & 1 & 1 & 1 & 1 & 1 & 0 & 0 & 0 \\
\hline 0 & 0 & 0 & 0 & 1 & 1 & 1 & 1 & 1 & 1 & 1 & 1 & 1 & 1 & 1 & 1 & 1 & 1 & 1 & 1 & 1 & 1 & 1 & 1 & 1 & 1 & 0 & 0 & 0 & 0 \\
\hline 0 & 0 & 0 & 0 & 0 & 1 & 1 & 1 & 1 & 1 & 1 & 1 & 1 & 1 & 1 & 1 & 1 & 1 & 1 & 1 & 1 & 1 & 1 & 1 & 1 & 0 & 0 & 0 & 0 & 0 \\
\hline 0 & 0 & 0 & 0 & 0 & 0 & 1 & 1 & 1 & 1 & 1 & 1 & 1 & 1 & 1 & 1 & 1 & 1 & 1 & 1 & 1 & 1 & 1 & 1 & 0 & 0 & 0 & 0 & 0 & 0 \\
\hline 0 & 0 & 0 & 0 & 0 & 0 & 0 & 1 & 1 & 1 & 1 & 1 & 1 & 1 & 1 & 1 & 1 & 1 & 1 & 1 & 1 & 1 & 1 & 0 & 0 & 0 & 0 & 0 & 0 & 0 \\
\hline 0 & 0 & 0 & 0 & 0 & 0 & 0 & 0 & 0 & 1 & 1 & 1 & 1 & 1 & 1 & 1 & 1 & 1 & 1 & 1 & 1 & 0 & 0 & 0 & 0 & 0 & 0 & 0 & 0 & 0 \\
\hline 0 & 0 & 0 & 0 & 0 & 0 & 0 & 0 & 0 & 0 & 0 & 1 & 1 & 1 & 1 & 1 & 1 & 1 & 1 & 0 & 0 & 0 & 0 & 0 & 0 & 0 & 0 & 0 & 0 & 0 \\
\hline 0 & 0 & 0 & 0 & 0 & 0 & 0 & 0 & 0 & 0 & 0 & 0 & 0 & 0 & 0 & 0 & 0 & 0 & 0 & 0 & 0 & 0 & 0 & 0 & 0 & 0 & 0 & 0 & 0 & 0 \\
\hline
\end{tabular}

Fig. 4. Mask image and mask coefficients

\section{RIDGELETS}

The basic difference between ridgelets and wavelets is that wavelets relate the scales of the point position and ridgelets relate the scales of the line positions as given by [8, 30]. Thus, wavelets are very good at representing point singularities. However, when it comes to line singularities wavelets fail and ridgelets are the solution, as demonstrated by [34]. The ridgelet transform of an image $f(x, y)$ is given by equations (4) and (5).

$$
\begin{gathered}
D f=\hat{f}(a, b, \theta)=\int_{R^{2}} \psi_{a, b, \theta}(x, y) f(x, y) d x d y \\
\psi_{a, b, \theta}(x, y)=a^{-1 / 2} \psi(((x, y) \cdot \xi-b) / a)
\end{gathered}
$$

Where, $D f$ is ridgelet transform, $a$ is scaling factor, $b$ is the shift, $\theta$ is the rotation and $\xi=(\cos \theta, \sin \theta)$. This can be computed by using the Radon and Wavelet transforms. In combination as described by (6) and (7).

$$
D f=W R f=\int_{R} \psi_{a, b}(t) \bar{f}(\theta, t) d t
$$




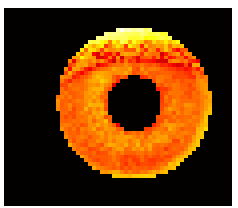

(1)

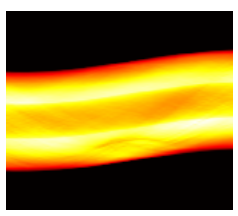

(2)

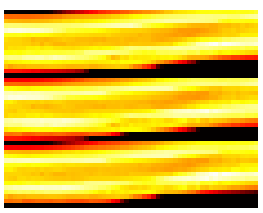

(3)

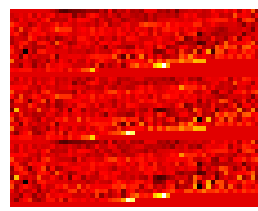

(4)

Fig. 5. Process for finding the ridgelet transform

$$
R f=\bar{f}(\theta, t)=\int_{R^{2}} f(x, y) \delta((x, y) \cdot \xi-t) d x d y
$$

A sample of a Ridgelet transform of an iris image is shown in Fig.s 5(3) and 5(4), which is obtained by a series of steps as shown in Figs. 5(1) and 5(2).

\subsection{The Radon Transform}

The Radon transform of an image is able to transform two-dimensional images with lines into a domain of possible line parameters, where each line in an image will give a peak that is positioned at the parameters of the corresponding lines. The most popular form of a radon transform expresses lines in the form given by equation (8).

$$
\rho=x \cos \theta+y \sin \theta
$$

Where, $\theta$ is the angle and $\rho$ is the smallest distance to the origin of the coordinate system. Thus, the radon transform for a set of parameters $(\rho, \theta)$ is the integral line through image $f(x, y)$, where the line is positioned corresponding to the value of $(\rho, \theta)$.

\subsection{1-D Wavelet Transform}

Computing the wavelet coefficients at every possible scale is a fair amount of work, and it generates an awful lot of data. That is why only a subset of scales and positions at which to make the calculations are considered. It turns out, rather remarkably, that if we choose scales and positions based on the powers of two so-called dyadic scales and positions, then the analysis will be much more efficient and accurate with a size that is reduced to $50 \%$. Also, factor two is the lowest possible scale, which is an integer. Such an analysis from the Discrete Wavelet Transform (DWT) is given by (9).

$$
D W T=\sum_{k=1}^{\infty} \sum_{l=-\infty}^{+\infty} q(k, l) \psi\left(2^{-k} t-l\right)
$$

An efficient way to implement this scheme using filters was developed in 1988. This algorithm is in fact a classical scheme known in the signal processing community as a two-channel sub band coder. This very practical filtering algorithm yields a fast wavelet transform. Equations (11) and (12) are used to compute wavelet functions. 


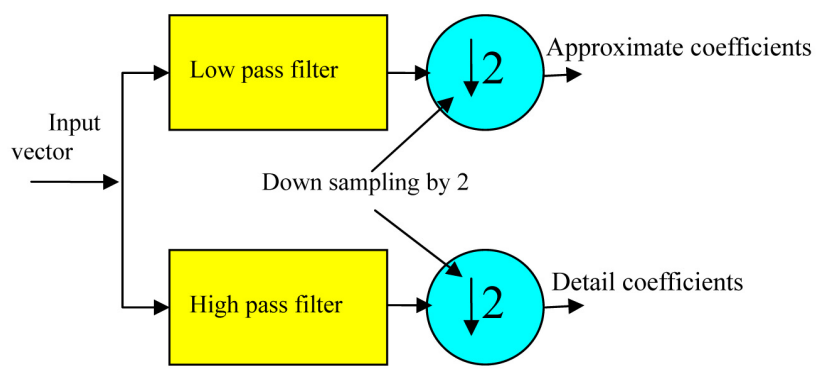

Fig. 6. Algorithm to compute a one level $1 \mathrm{D}$-wavelet transform

$$
\begin{array}{r}
\phi(x)=\sum_{n} h_{\phi}(n) \sqrt{2} \phi(2 x-n) \\
\psi(x)=\sum_{n} h_{\psi}(n) \sqrt{2} \psi(2 x-n)
\end{array}
$$

Both the $\phi(x)$ scaling function and $\psi(x)$ wavelet function can be expressed as linear combinations of double-resolution copies of themselves. Here $h_{\varnothing}$ in (10) and $h_{\psi}$ in (11) are the expansion coefficients and are called scaling and wavelet vectors, respectively. They are the filter coefficients of the Fast Wavelet Transform (FWT).

The algorithm shown in Fig. 6 can obtain single level one-dimensional wavelet decomposition.

\section{Proposed Method}

The feature vector size used by the proposed method is very small and is $1 \mathrm{X} 4$ in size as compared to the signature size used by [20], which also uses iris normalization. The proposed method skips this normalization. It uses the ridgelet transform for feature extraction. Line singularities of an iris are obtained by using ridgelets. The proposed method is discussed in detail in the following sections.

\subsection{Image database}

The database used in this work is both the Chinese Academy of Sciences-Institute of Automation-CASIA (Version 1 and Version 3) database and the UPOL database [42-48]. The Chinese Version 1 database used in the experimentation consists of 756 different iris images from the CASIA iris image database [7]. The CASIA iris database is a public domain database. The size of each iris image in the database is $280 \times 320$ in Version 1. In this database, there are 108 different subjects. Each subject has contributed to seven different images of the same eye. Thus, there are $756(108 \times 7)$ images in the database. Among the seven images, three images were taken in the first session and the other four were taken in the second session. Other databases like Cassia Version 3 and UPOL are also used. CASIA Iris Version 3 Intervals database though 
consist of 249 subjects and the authors could use only 33 subjects. Only 33 subjects in the data base were to be found with 20 images each. Each subject contributed 20 eye images. Among them 10 are of the left eye the other 10 are of the right eye. The size of each iris image in this database is also $280 \times 320$, as in Version 1 . The UPOL iris database consists of 384 different iris images. The size of each database iris image is $576 \times 768 \times 3$. In this database there are 64 different subjects. Each subject has contributed towards 6 images- 3 of the left eye and another 3 of the right eye.

\subsection{Feature database creation}

Using the masks that were designed each of the eye image in the database is filtered. Subsequently, every filtered image is decomposed to three levels using wavelets. The results obtained with normalization and those obtained without normalization using different masks are compared with each other.

To extract the signature of the iris we have computed the first level energy by using equation (12) and the standard deviation feature by equation (13) of each sub-band. Thus the feature vector database is $756 \mathrm{X} 24$ in size and the feature vector of the query image is $1 \mathrm{X} 24$ in size. The first level energy is computed by equation (12).

$$
\text { Energy }=E_{k}=\sum_{m=0}^{M-1} \sum_{n=0}^{N-1}|X(m, n)|
$$

Where,

$X(m, n)$ is a discrete function whose energy is to be computed.

Similarly standard deviation is given by equation (13).

$$
\sigma_{k}=\left(\frac{1}{M X N}\right) \sum_{i=1}^{N} \sum_{j=1}^{N} E\left[W_{k}(i, j)-\mu_{k}\right]
$$

Where,

$W_{k}(i, j)$ is the $\mathrm{k}^{\text {th }}$ wavelet decomposed sub-band.

$M X N$ is the size of wavelet decomposed sub-band.

$\mu_{k}$ is the mean value of $\mathrm{k}^{\text {th }}$ decomposed sub-band. The feature vector is given by equation (14).

$$
\mathrm{f}_{\mathrm{k}}=\left\lfloor\mathrm{E}_{\mathrm{k}}, \sigma_{\mathrm{k}}, \ldots \ldots \ldots \ldots \ldots \ldots \ldots \ldots \ldots \ldots\right.
$$

\subsection{Matching}

A query signature is one of the 756 signatures from the CASIA Version 1 image database signatures. It is one of the iris signature from among the 660 CASIA Version 3 databases or one of the iris signature from among the 384 for the UPOL database. The Euclidean distance metric given by (15) is used to compute the similarity or match value for the given pair of signatures in the corresponding database. "Zero distance" implies a perfect match, and the signature tends towards a mismatch as the distance increases. 


$$
D_{(x, y)}^{E u c l i}=\sqrt{\sum_{i=0}^{N}\left(x_{i}-y_{i}\right)^{2}}
$$

Where,

$x_{i}$ is the $\mathrm{i}^{\text {th }}$ element of $\mathrm{x}^{\text {th }}$ database iris signature and $y_{i}$ is the $\mathrm{i}^{\text {th }}$ element of the $\mathrm{y}^{\text {th }}$ query iris signature vector.

\subsection{Performance measures}

The threshold value used is the average distance of the top seven in CASIA 1, 10 in CASIA 3, and 3 in the UPOL signatures as they belong to different eye images of the same person's eyes. The threshold set is used to compute the False Acceptance Rate (FAR) and False Rejection Rate (FRR). Here the FAR and FRR are the performance measures used. Also, the Receiver Operating Curves (ROC) is another performance evaluation measure used.

\subsubsection{False Acceptance Rate (FAR)}

The FAR is also known as a Type I error. It is computed by (16)

$$
F A R=\frac{N F A}{N I V A}
$$

Where,

NFA is the Number of False Acceptances.

NIVA is the Number of Impostor Verification Attempts.

The FAR is the measure of likelihood that the biometric security system would incorrectly accept an attempt by an unauthorized user to access the system.

\subsubsection{False Rejection Rate (FRR)}

The FRR is also known as a Type II error. It is computed by (17).

$$
F R R=\frac{N F R}{N E V A}
$$

Where,

NFR is the Number of False Rejections.

NEVA is the Number of Enrollee Verification Attempts.

The FRR is the measure of likelihood that the biometric security system would incorrectly reject an attempt by an authorized user denying the access of the system.

\subsubsection{Average Accuracy Rate}

This gives the average accurate recognition. Equation (18) can compute it.

$$
\text { Accuracy rate }=\left(1-\left(\frac{F A R+F R R}{100}\right)\right) * 100
$$

Where, FAR is the False Acceptance Rate and FRR is the False Rejection Rate 


\subsubsection{Receivers Operating Characteristics (ROCs):}

The ROC is a plot of the Genuine Acceptance Rate against the False Acceptance Rate for all possible system-operating points (that is, the matching threshold) and it measures the overall performance of the system. Each point on the curve represents a particular decision threshold. In an ideal case, both the error rates that are the FAR and FRR should be zero and the imposter distribution should be disjointed. The ideal ROC in such a case is a step function at zero FAR. On the other hand, if the genuine and imposter distributions are exactly the same, then the ROC is a line segment with a slope of $45^{\circ}$ with an end point at zero FAR. In practice, these curves behave in between these two extremes. An Equal Error Rate (ERR) is defined as the operating point where the two types of errors (FAR and FRR) are equal. The proposed method is also compared with Gabor filters, which uses a $600 \%$ larger signature than the ridgelets. The ROC curves are as shown in Fig. 7. The range of the False Acceptance Rate is a very small magnified view of the ROC and is given in Fig. 8.

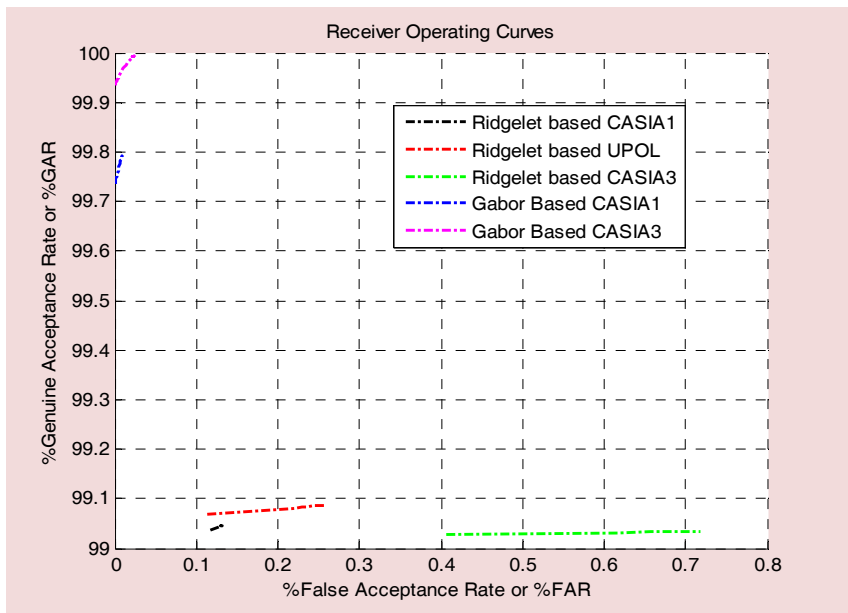

Fig. 7. ROC curves

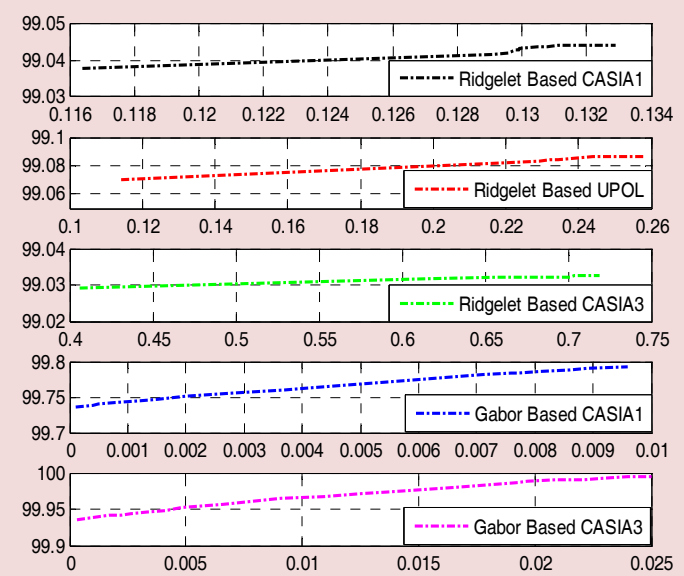

Fig. 8. Magnified view of ROC Curves 


\section{RESULtS}

The results obtained by the proposed method are very interesting and reliable. They are listed below in Table 1.

Table 1 gives a comparison of different algorithms and the proposed method. It is evident from this table that the results obtained by the proposed method are comparatively much better than the existing methods, though we have used a signature of a very small size that is just 1X4 . The highest accuracy obtained is $99.82 \%$. No doubt that the accuracy obtained by Daugman's method is $99.90 \%$, but the signature size is 1 X2048 (phase) bit vector. The Gabor based method is $600 \%$ computationally more expensive in terms of signature size. Thus, it is evident that the results are comparatively outperforming other methods in terms of all the performance measures like iris signature size, False Acceptance Rate (FAR), False Rejection Rate (FRR), and accuracy.

Table 1. Comparison of the proposed method with existing methods

\begin{tabular}{|c|c|c|c|c|c|}
\hline \multicolumn{2}{|l|}{ Algorithm } & Feature vector length & FAR & FRR & Accuracy $(\%)$ \\
\hline \multicolumn{2}{|c|}{ Daugman [6] } & 1X2048 (bits) & 0.0100 & 0.0900 & 99.90 \\
\hline \multicolumn{2}{|c|}{ Tisse et al. [15] } & $\begin{array}{l}-- \\
-\end{array}$ & 1.8400 & 8.7900 & 89.37 \\
\hline \multicolumn{2}{|c|}{ Ma et al. [20] } & ---- & 0.0200 & 1.9800 & 98.00 \\
\hline \multicolumn{2}{|c|}{ Reillo et al. [21] } & --- & 0.0300 & 2.0800 & 97.89 \\
\hline \multicolumn{2}{|c|}{ Cui et al. [19] } & --- & 1.2000 & 31.500 & - \\
\hline \multicolumn{2}{|c|}{ Chu and Chen [14] } & --- & 0.0000 & 0.6900 & 99.14 \\
\hline \multicolumn{2}{|c|}{ Maheswari et al. [29] } & --- & 0.0100 & 0.8800 & - \\
\hline \multirow{2}{*}{ Gabor based } & CASIA Ver. 1 & $1 \times 24$ & 0.0046 & 0.2352 & 99.76 \\
\hline & CASIA Ver. 3 & $1 \mathrm{X} 24$ & 0.0121 & 0.0320 & 99.95 \\
\hline \multirow{3}{*}{ Ridgelets based (proposed) } & CASIA Ver.1 & $1 \mathrm{X} 4$ & 0.1309 & 0.0434 & 99.82 \\
\hline & CASIA Ver. 3 & $1 \mathrm{X} 4$ & 0.6641 & 0.0321 & 99.30 \\
\hline & UPOL & $1 \mathrm{X} 4$ & 0.2434 & 0.0852 & 99.67 \\
\hline
\end{tabular}

\section{CONCLUSION}

The proposed method introduces ridgelet to the field of iris recognition systems. It exploits the property of ridgelets to detect line singularities. From the listed tables it can be concluded that the proposed method provides improvement in almost all of the performance measures. This approach has a 3-fold advantage. Initially, the computational complexity is reduced. Secondly, the accuracy is improved and finally, the signature size is tremendously reduced to just $1 \mathrm{X} 4$. The high accuracy obtained by traditional methods is at the cost of system speed and signature size. But, the reduced iris signature by the proposed method does not affect the average accuracy at all. The iris signature is comparatively small at $1 \mathrm{X} 4$ and it improves the system speed of an online system significantly. Though Daugman's method gives the best accuracy of $99.82 \%$, the iris signature length used by that algorithm is comparatively very high with a 1 X2048 bit vector. The proposed method gives an accuracy of $99.82 \%$, with a signature of a comparatively very small length. The proposed method also achieves computational inexpensiveness, as the Gabor filter based method is 600 times more expensive than the proposed method in terms of signature size.

The future scope of research is to obtain the highest accuracy with a zero False Acceptance Rate and False Rejection Rate. The proposed method can be extended to achieve zero FAR and 
zero FRR by increasing the levels of decomposition. However, this would cost in terms of signature size.

\section{REFERENCES}

[1] A. Muron and J. Pospisil, "The Human Iris Structure and its usages," In: Acta Univ, Palacki, Olomuc, Fac, Rer, Nat, 2000, Physica 39, pp.87-95.

[2] H. Salami and M.J.E Wahyudi, "Iris Recognition System by Using Support Vector Machines", International Conference on Computer and Communication Engineering, ICCCE, 13-15 May, 2008, pp.516-521.

[3] N .Barzegar and M.S Moin, "A new approach for iris localization in iris recognition systems, Computer Systems and Applications", AICCSA 2008, IEEE/ACS International Conference, March 31April, 4 2008, pp.516- 523.

[4] B. Faisal, C. Pablo, U. David and F. Marc, "Eagle-Eyes: A System for Iris Recognition at a Distance, Technologies for Homeland Security”, IEEE Conference, 12-13 May, 2008, pp.426-431.

[5] B. Faisal, U. David, C. Pablo and F. Marc, "Video Surveillance for Biometrics: Long-Range Multibiometric System, Advanced Video and Signal Based Surveillance, AVSS", IEEE Fifth International Conference, 1-3 September, 2008, pp.175-182.

[6] J. Daugman, "High Confidence Visual Recognition of Persons by a Test of Analysis of Statistical Independence," IEEE Trans on Pattern Analysis and Machine Intelligence, Vol.15. No.11, 1993, pp.1148-1161.

[7] J. Daugman, "Statistical Richness of Visual Information: Update on Recognising persons by Iris Patterns," International Journal on Computer Vision, Vol.45, No.1, 2001, pp.25-38.

[8] O.R. Terrades and E. Valveny, "Local Norm Features Based on Ridgelets Transform," Document Analysis and Recognition, Proceedings of Eighth International Conference, 2005, pp.700-704.

[9] L. Birgale, M. Kokare and D. Doye, "Color and Texture Features for Content Based Image Retrieval," Proceedings of 3rd IEEE International Conference CGIV, Sydney, Australia, August, 2006, pp.146-149.

[10] L. Birgale and M. Kokare, "Iris Recognition Using Discrete Wavelet Transform," Proceedings of IEEE International Conference ICDIP, Bangkok, Thailand, March, 7-9, 2009, pp.401-404.

[11] L. Birgale and M. Kokare, "Iris Recognition Without Iris Normalisation", Proceedings of IEEE National Conference, Indian Conference on Computer Vision, Graphics, Image and Video Processing, ICCVGIVP, Nagpur, India, March, 13-14, 2009, pp.249-251.

[12] L. Birgale and M. Kokare, "Iris Recognition Without Iris Normalisation," Journal of Computer Science, Vol.6, No.9, Science publications, 2010, pp.1042-1047.

[13] L. Birgale and M. Kokare, "A Survey on Iris Recognition", The Icfai University Journal of Electrical \& Electronics Engineering (IUJEEE), Vol.2, No.4, October, 2009, pp.7-25.

[14] C. Chu and H. Chen, "High Performance Iris Recognition Based on LDA and LPCC", IEEE. Proceedings of Conference on Tools with Artificial Intelligence (ICTAI), 2005, pp.9-13.

[15] L. Tisse, C.Loic, M. Torres and M. Robert, "Person Identification based on iris patterns", Proceedings of the 15th International Conference on Vision interface, 2002, pp.215-129.

[16] D. Hasan and A. Gholamreza, "Iris recognition system using combined histogram statistics," Computer and Information Sciences, ISCIS, 23rd International Symposium, 27-29 October, 2008, pp.1- 4.

[17] E. Rydgren, E.A. Thomas, F. Amiel, F. Rossant and A. Amara, "Iris Features Extraction using wavelet Packets", IEEE Transaction, an International Conference on Image Processing (ICIP), 2004, pp.861-864.

[18] L. Flom and A. Safir: Iris recognition system, U.S Patent No.4. 641349. Washinton, U.S. Government, 1994.

[19] J. Cui, Y. Wang, J. Zhou, H. Tieniu and T. Zhenan, “An Iris Image Synthesis Method Based on PCA and Super-resolution", Proceedings of the 17th International Conference on Pattern Recognition (ICPR), 2004, pp.147-152.

[20] L. Ma, Y. Wrag and T.Tam, "Iris Recognition Using Circular Symmetric Filters," Pattern Recogni- 
tion, Vol.2, No.2, pp.414-417.

[21] R.S. Reillo, C.S. Avila, D. Martin and D. Roche, "Iris Recognition for Biometric Identification using Dyadic Wavelet Transform Zero Crossing", Proceedings of the IEEE 35th Carnahan International Conference on Security Technology, 2002, pp.272-277.

[22] R.P. Wildes, J.C. Asmultr, G.L, Green, S.C, Hsu, R.J. Kolczynski, J.R. Matey and S.E. McBride, "A Machine Vision System for Iris Recognition," Machine Vision and Application, Vol.9, No.2, 1996, pp.1-8.

[23] Y. Richard, N. Fatt, H. Yong, K. Tay and M. Ming, "Iris Recognition Algorithms Based on Texture Analysis," Information Technology, ITSim, International Symposium, 26-28 August, 2008, pp.1-5.

[24] Tajbakhsh, N. Araabi, N. Babak and S. Hamid, "An Intelligent Decision Combiner Applied to Noncooperative Iris Recognition”, Information Fusion, 11th International Conference, June 30-July 3, 2008, pp.1 -6.

[25] P.T. Gonzalez, A.F. Fernandez and O.J. Garcia, "On the Effects of Time Variability in Iris Recognition", Biometrics: Theory, Applications and Systems, BTAS, 2nd IEEE International Conference. 29 September-1 October, 2008, pp.1-6.

[26] Y. Xueyi, H. Zhiwei and Z. Wencong, "A Data Hiding Method for Improving the Self-Security of Iris Recognition", International Conference on Communications, Circuits and Systems, ICCCAS, 25-27 May, 2008, pp.762-768.

[27] N. Boddeti, B.V. Kumar and K. Vijaya, "Extended Depth of Field Iris Recognition with Correlation Filters", Biometrics: Theory, Applications and Systems, BTAS, 2nd IEEE International Conference, Sept. 29-Oct. 1 2008, pp.1-8.

[28] J. Lu and M. Xie, "Use fractional calculus in iris localization", International Conference on Communications Circuits and Systems, ICCCAS, 25-27 May, 2008, pp.946-949.

[29] M. Maheswari, P. Anbalagan and T. Priya, "Efficient Iris Recognition through Improvement in Iris Segmentation Algorithm," ICGST-GVIP Journal. ISSN: 1687-398X, Vol.8, No.2, 2008, pp.29-35.

[30] Minh, N. Do, Martin, Vetterli. (2003) "The Finite Ridgelet transform for Image Representation", IEEE Trans. On Image Processing. Vol.12, pp.16-28.

[31] M. Taihei, L. Yung-hui and S. Marios, "Hallucinating Irises-Dealing with Partial and Occluded Iris Regions", Biometrics: Theory, Applications and Systems, BTAS, 2nd IEEE International Conference, Sept. 29 October, 2008, pp.1-6.

[32] N.Richard, Y.Fatt, T. Yong, H. Mok and K. Ming "A review of iris recognition algorithms," Information Technology, ITSim, International Symposium, Vol.2, 26-28 August, 2008, pp.1-7.

[33] E.M. Newton and P.J. Phillips, "Meta-Analysis of Third-Party Evaluations of Iris Recognition," Systems, Man and Cybernetics, Part A, IEEE Trans, Vol.39, No.1, January, 2009, pp.4-11.

[34] C. Philippe and A. Eric, "Discrete Analytical Ridgelet Transform," Signal Processing, Vol.11, No.84, 2004, pp.2165-73.

[35] P. Polash, P. Maruf and M. Monwar, "Human iris recognition for biometric identification", Computer and Information Technology, ICCIT, 10th International Conference, 27-29 December, 2007, pp.1-5.

[36] Q. Chuan and Tian, "A New Iris Region Segmentation Method", Fuzzy Systems and Knowledge Discovery, FSKD, Fifth International Conference, 18-20 October, 2008, pp.63-67.

[37] R. Youmaran, L.P. Xie and A. Adler, "Improved Identification of Iris and Eyelash Features", Communications, 24th Biennial Symposium, 24-26 June, 2008, pp.387-390.

[38] Y.Shiun, C. Lee, C. Lin, Huang, M. Fen, Ho, W. Liang, and Huang, "Iris recognition using Dynamic Programming Matching Pursuit", International Conference on Audio Language and Image Processing, ICALIP, 7-9 July, 2008, pp.1471-1476.

[39] Z. Li, X. Zhu and Y.Wang, "A Study on Designing Iris Recognition System Based on TMS320DM642", Networking, Sensing and Control, ICNSC, IEEE International Conference, 6-8 April, 2008, pp.13951399.

[40] Z. Sheikh, D.N. Matthew, "Iris Recognition Performance Enhancement using Weighted Majority Voting", Image Processing, ICIP, 15th IEEE International Conference, 12-15 October, 2008, pp.277-280.

[41] Z. Jinyu and S.A. Natalia, "An Automatic Algorithm for Evaluating the Precision of Iris Segmentation", Biometrics: Theory, Applications and Systems, BTAS, 2nd IEEE International Conference, 
September, 29, 2008, pp.1-6.

[42] Chinese Academy of Sciences-Institute of Automation. Database of 756 Greyscale Eye Images [online database]. "http://www.sinobiometrics.com" Version 1.0, 2003.

[43] M. Dobes, J. Martinek, D. Skoupil, Z. Dobesova and J. Pospisil, "Human Eye Localization using the Modified Hough Transform", Optik, Vol.117, no.10, Elsevier 2006, pp.468-473.

[44] M. Dobes, L Machala, P. Tichavsky and J. Pospisil, "Human Eye Iris Recognition Using the Mutual Information", Optik Vol.115, No.9, Elsevier 2004, pp.399-405.

[45] M. Dobes and L. Machala, Iris Database, [Online database]. Available: "http://www.inf.upol.cz/iris/"

[46] W. Zhifang, H. Qi, N. Xiamu and B. Christoph, "A Novel Template Protection Algorithm for Iris Recognition", Intelligent Systems Design and Applications, ISDA, Eighth International Conference, 26-28 November, 2008, pp.340-345.

[47] F. Wheeler, W. Perera, A.G. Amitha, A. Gil, Y. Bing and T.H. Peter, "Stand-off Iris Recognition System", Biometrics: Theory, Applications and Systems, 2nd IEEE International Conference, 29 September-1 October, 2008, pp.1-7.

[48] Y. Zhu, T. Tan and Yusag, "Biometric Personal Identification based on Iris Patterns," Pattern Recognition, Vol.2, No.2, 2004, pp.801-804.

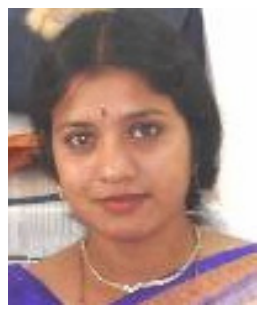

\section{Ms. Lenina Birgale}

She received her B.E. and M. E. Degree in Electronics Engineering from Shri Guru Gobind Singhij Institute of Engineering and Technology (SGGSIE\&T) Nanded in Maharashtra, India, in 2001 and 2005, respectively. She submitted her doctoral thesis to Swami Ramanand Teerth Marathawada University (SRTMU) Nanded in Maharashtra, India, in August 2011. She has been in academics in the Department of Electronics and Telecommunication Engineering since 2001. Currently, she is an Assistant Professor at Shri Guru Gobind Singhji Institute of Engineering and Technology (SGGSIE\&T) Nanded in Maharashtra, India, in the Department of Electronics and Telecommunication Engineering. She is a lifetime member of the Indian Society for Technical Education.

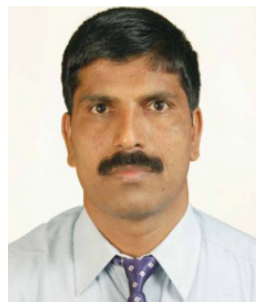

\section{Dr. Manesh Kokare}

He received his Diploma in Industrial Electronics Engineering from the Board of Technical Examination in Maharashtra, India, in 1990. He received his B.E. and M.E. Degree in Electronics Engineering from Shri Guru Gobind Singhij Institute of Engineering and Technology (SGGSIE\&T) Nanded in Maharashtra, India, in 1993 and 1999 respectively, and his Ph.D. from the Department of ECE at the Indian Institute of Technology in Kharagpur, India, in 2005. From June 1993 to Oct. 1995, he worked with industry (Superb Products Pvt Ltd, Pune as a Trainee Engineer and also with Desai Electronics Pvt Ltd, Pune as a Junior Engineer). From Oct. 1995, he started his career in academics in the Department of Electronics and Telecommunication Engineering at SGGSIE\&T Nanded, where he is presently holding the position of an Associate Professor. He has published more than 55 papers in international and national journals and conferences. He received the prestigious Career Award for Young Teachers (CAYT) for the year 2005 from the All India Council for Technical Education (AICTE) in New Delhi, India. In December 2009, he was honored with the "Best Achiever of SGGSIE\&T Alumni" award. Recently Dr. Kokare has been awarded the "BOYSCAST" Fellowship for 2010-2011 by the Department of Science and Technology from the government of India to carry out his postdoctoral research work at the University of California Santa Barbara in the USA. He is a lifetime member of the System Society of India, the Indian Society for Technical Education, and the Institution of Electronics and Telecommunication Engineers, and is also a member of the IEEE. 